\title{
IBX-mediated oxidation of unactivated cyclic amines: application in highly diastereoselective oxidative Ugi-type and aza-Friedel-Crafts reactions $\uparrow$
}

Cite this: Org. Biomol. Chem., 2015, 13, 10108

Received 23rd July 2015,

Accepted 7th September 2015

DOI: $10.1039 /$ c5ob01519g

www.rsc.org/obc

The first o-iodoxybenzoic acid (IBX) mediated oxidation of unactivated amines to imines is described. A range of meso-pyrrolidines were shown to be suitable substrates. The chemical space was further explored with one-pot oxidative Ugi-type and aza-Friedel-Crafts reactions, which proved to be highly diastereoselective.

The chemistry of hypervalent iodine reagents (Fig. 1) has received major interest in recent years as a result of the increasing number of new reagents and their application in diverse chemical transformations. ${ }^{1}$ In particular, $o$-iodoxybenzoic acid (IBX) ${ }^{2}$ has experienced increasing attention owing to its broad applicability and high chemoselectivity. Santagostino et al. developed a convenient and cost-efficient synthesis of high purity IBX by the treatment of 2-iodobenzoic acid with oxone in aqueous medium, ${ }^{3}$ which renders IBX an easily accessible reagent. Chemical transformations mediated by IBX

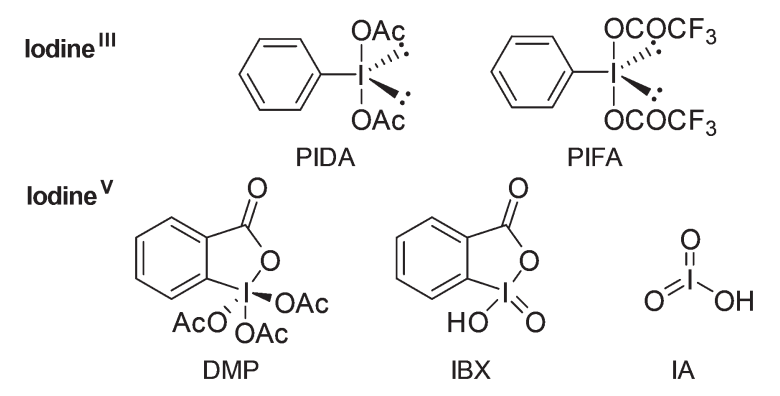

Fig. 1 A selection of commonly used and commercially available hypervalent iodine reagents and their acronyms.

Department of Chemistry \& Pharmaceutical Sciences and Amsterdam Institute for Molecules Medicines and Systems (AIMMS), VU University Amsterdam, De Boelelaan 1083, 1081 HVAmsterdam, The Netherlands.E-mail: r.v.a.orru@vu.nl

$\dagger$ Electronic supplementary information (ESI) available: Optimization data, experimental procedures, compound characterization data, copies of ${ }^{1} \mathrm{H}$ and ${ }^{13} \mathrm{C}$ spectra, and X-ray crystallographic data of compound 3i. CCDC 1400648. For ESI and crystallographic data in CIF or other electronic format see DOI: 10.1039/ c5ob01519g include the selective oxidation of primary and secondary alcohols to the corresponding aldehydes and ketones as well as dehydrogenation of aldehydes and ketones to $\alpha, \beta$-unsaturated carbonyl compounds. Nicolaou et al. described the oxidation of benzylic amines as well as amines that aromatize upon oxidation, ${ }^{4,5}$ however, the oxidation of unactivated aliphatic amines with IBX was not investigated. ${ }^{6}$

The direct $\alpha$-functionalization of amines is a highly interesting transformation that attracted great attention in recent years. $^{7}$ Consequently, transition metal-catalyzed oxidative versions of important $\mathrm{C}-\mathrm{C}$ bond-forming reactions such as Mannich, Strecker, aza-Henry and aza-Friedel-Crafts reactions have been developed. ${ }^{8}$ A limited number of examples of the application of IBX in oxidative multicomponent reactions has been described, i.e. oxidative Strecker, ${ }^{9}$ Passerini, ${ }^{10}$ Ugi and Ugi-type ${ }^{11}$ reactions. Although unactivated alcohols were suitable substrates, the scope of the amine component is basically unexplored with the exception of a small selection of $\alpha$-activated amines, such as benzylic amines, $\alpha$-amino nitriles and $\alpha$-amino esters.

Herein, we report a mild and selective oxidation of unactivated cyclic amines with IBX to access the corresponding imines. The oxidation can be combined in one pot with the addition of $C$-nucleophiles, providing oxidative Ugi-type and aza-Friedel-Crafts reactions.

In light of our continued interest in the functionalization of imines, in particular 1-pyrrolines, ${ }^{12}$ we envisioned a clean and fast oxidation of unactivated meso-pyrrolidines. Examination of the currently available methods revealed that the oxidation of unactivated secondary amines is rather challenging. ${ }^{13}$ Given the recent regained interest in hypervalent iodine reagents, we decided to explore their ability to oxidize aliphatic amines. We started our investigation with the oxidation of meso-pyrrolidine 1a with different commercially available hypervalent iodine reagents at room temperature in the typically used solvent DMSO (Table 1, entries 1-4). ${ }^{4}$ The desired 1-pyrroline (2a) was obtained in high yield (Table 1 , entry 4) with only one equivalent of IBX, while oxidants such as (diacetoxy)iodobenzene (PIDA), [bis(trifluoroacetoxy)]iodobenzene (PIFA) and Dess-Martin 
Table 1 Optimization of reaction conditions for the oxidation of mesopyrrolidine $1 a^{a}$

\begin{tabular}{llll}
\hline & & \\
\hline Entry & Oxidant & Conditions & Yield $^{b}(\%)$ \\
\hline 1 & PIDA & DMSO, RT, $30 \mathrm{~min}$ & 38 \\
2 & $\mathrm{PIFA}$ & $\mathrm{DMSO}, \mathrm{RT}, 30 \mathrm{~min}$ & 33 \\
3 & $\mathrm{DMP}$ & $\mathrm{DMSO}_{\mathrm{RT}}, 30 \mathrm{~min}$ & 55 \\
4 & $\mathrm{IBX}$ & $\mathrm{DMSO}_{\mathrm{RT}}, 30 \mathrm{~min}$ & 90 \\
$5^{c}$ & $\mathrm{IBX}$ & $\mathrm{CH}_{2} \mathrm{Cl}, 60{ }^{\circ} \mathrm{C}, 1 \mathrm{~h}$ & 95
\end{tabular}

${ }^{a}$ Conditions: amine $(0.25 \mathrm{mmol})$, oxidant $(1 \mathrm{eq}$.$) , solvent (0.2 \mathrm{M})$. ${ }^{b}$ Yield determined by adding 2,5-dimethylfuran (0.5 eq.) after the reaction workup as standard for NMR spectroscopy. ${ }^{c}$ Closed vessel.

periodinane (DMP) gave poor to moderate yields (Table 1 , entries 1-3). A solvent screen revealed that the oxidation proceeds well in a range of protic as well as aprotic solvents in which IBX is virtually insoluble (see ESI $\dagger$ ), although an increased temperature and reaction time were required $\left(60{ }^{\circ} \mathrm{C}\right.$, $1 \mathrm{~h}$ ). Superior results were obtained using $\mathrm{CH}_{2} \mathrm{Cl}_{2}$ (Table 1, entry 5), ${ }^{14}$ which prompted us to select this solvent for a screening of the substrate scope.

To our delight, a range of aliphatic meso-pyrrolidines was selectively oxidized by IBX towards the corresponding 1-pyrrolines in good to excellent yield (Table 2, 70-97\%). Hemiaminal 2f is a highly interesting building block for the synthesis of aza-sugars, but was obtained in moderate yield presumably as a result of instability due to its increased electrophilicity. ${ }^{15}$ Under the same conditions, reactions of monocyclic pyrrolidines gave mixtures of the corresponding pyrrolines and pyrroles. ${ }^{16}$ We hypothesize that this overoxidation is caused by tautomerization of the initially formed imines to the corresponding enamines, which subsequently undergo a second oxidation leading to pyrroles. In case of bi- and tricyclic 1-pyrrolines $\mathbf{2 a - f ,}$ tautomerization is prevented and overoxidation does not occur.

Imines are interesting inputs for a wide variety of complexity-generating reactions as a result of their electrophilic as well as nucleophilic properties. For example, imines serve as templates for many multicomponent reactions (MCRs), a category of reactions that create a high degree of diversity and complexity in a single step. ${ }^{17}$ One of the most studied and widely applied MCRs is the Ugi reaction, which has proven to be a powerful tool for rapid synthesis of lead compounds in drug discovery. ${ }^{18}$ Consequently, we envisioned a diastereoselective oxidative Ugi-type three-component reaction for the synthesis of $\mathrm{N}$-acylprolyl amide derivatives. ${ }^{19}$

For a screening of the reaction conditions, the reaction between meso-pyrrolidine 1a, benzoic acid and tert-butyl isocyanide was investigated (Table 3 ). In agreement with earlier
Table 2 Scope study for oxidation of meso-pyrrolidines 1 with IBX

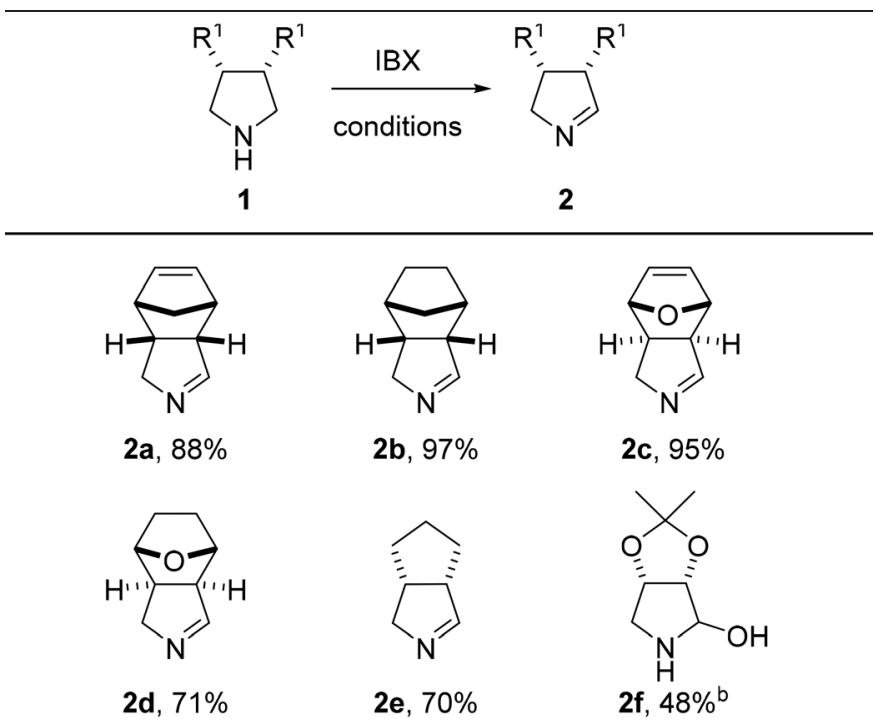

${ }^{a}$ Conditions: amine ( $\left.0.25 \mathrm{mmol}\right)$, IBX (1 eq.), DCM $(0.2 \mathrm{M}), 60^{\circ} \mathrm{C}, 1 \mathrm{~h}$, closed vessel. Isolated yield, unless stated otherwise. ${ }^{b}$ Yield determined by adding 2,5-dimethylfuran ( 0.5 eq.) after the reaction workup as standard for NMR spectroscopy and proposed structure (2f) was not fully characterized (see ESI). MeCN as solvent.

Table 3 Optimization of reaction conditions for the oxidative Ugi-type three-component reaction ${ }^{a}$

\begin{tabular}{lll}
\hline \\
Entry
\end{tabular}

${ }^{a}$ Conditions: amine $(0.25 \mathrm{mmol})$, benzoic acid (1.5 eq.), tert-butyl isocyanide (1.5 eq.), IBX (1 eq.), solvent $(0.5 \mathrm{M}), 60^{\circ} \mathrm{C}, 24 \mathrm{~h}$, closed vessel. ${ }^{b}$ Yield determined by adding 2,5 -dimethylfuran ( 0.5 eq.) after the reaction workup as standard for NMR spectroscopy.

reports, ${ }^{10,11}$ a high concentration of reagents proved to be beneficial for the reaction outcome (see ESI $\dagger$ ). The solvent of choice for most Ugi reactions, $\mathrm{MeOH}$, was used as reaction medium with modest efficiency (Table 3, entry 1). Competing oxidation of the solvent could pose a problem, although previous reports suggest that the oxidation of amines is generally much faster than alcohols. ${ }^{4 a, b, 11 b}$ Considering the complexity of the reaction, satisfactory results were obtained using either MeCN or $\mathrm{CH}_{2} \mathrm{Cl}_{2}$ as the solvent (Table 3, entries 2 and 3). As a consequence of the superior results obtained for the oxidation 
of a variety of meso-pyrrolidines in $\mathrm{CH}_{2} \mathrm{Cl}_{2}$, this solvent was selected for investigation of the reaction scope. The one-pot reaction could be performed with a range of meso-pyrrolidines, affording the corresponding dipeptides in modest to good yields (Table 4, 41-61\%). A range of electronically diverse carboxylic acids were suitable reaction partners, although it should be noted that lower yields were observed for aliphatic acids such as acetic acid (3e, 43\%). Various aliphatic and aromatic isocyanides were compatible with the oxidative Ugi-type reaction as well. Although hemiaminal $2 \mathrm{f}$ was found to be unstable upon isolation, Ugi adduct $\mathbf{3 h}$ could be obtained in $45 \%$ yield as a single diastereoisomer. Notably, 3,3-dimethylindoline - with blocked benzylic positions to prevent benzylic oxidation $^{20}$ - could also be applied with modest efficiency $(3 \mathbf{j}$, $35 \%) .^{21}$

Table 4 Oxidative Ugi-type three-component reaction ${ }^{a}$
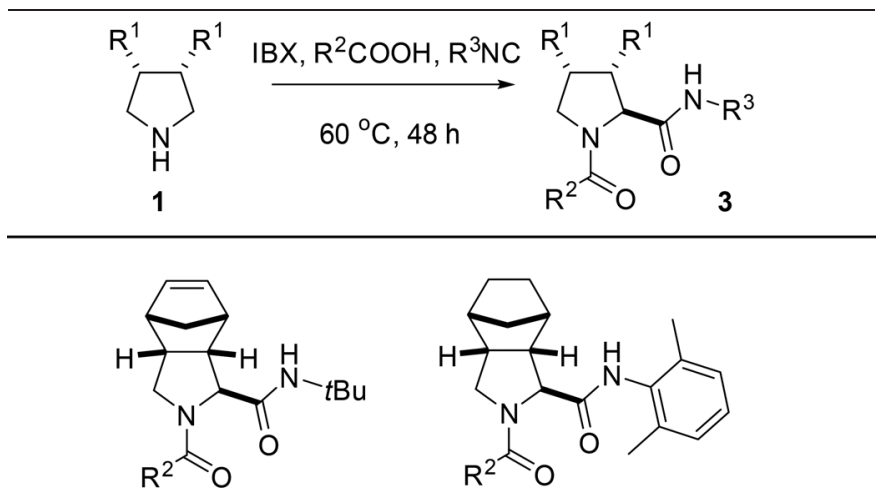

3a, $R^{2}=\mathrm{Ph}, 59 \%$
3 b, $\mathrm{R}^{2}=p-\mathrm{NO}_{2} \mathrm{C}_{6} \mathrm{H}_{4}, 52 \%$
3 c, $\mathrm{R}^{2}=\mathrm{PMB}, 56 \%$

\begin{abstract}
3d, $\mathrm{R}^{2}=\mathrm{Ph}, 61 \%$
\end{abstract}
3e, $R^{2}=M e, 43 \%$

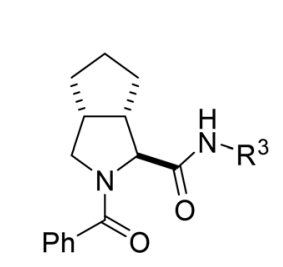

3f, $\mathrm{R}^{3}=\mathrm{cHex}, 54 \%$

$3 g, R^{3}=B n, 41 \%$<smiles>COc1cc(CNC(=O)[C@@H]2[C@H]3OC(C)(C)O[C@@H]3CN2C(=O)/C=C/c2ccccc2)cc(OC)c1</smiles><smiles>CC(C)(C)NC(=O)[C@@H]1[C@H]2C=C[C@H](O2)[C@H]1CN(C(=O)c1ccccc1)C(C)(C)C</smiles>

$3 \mathbf{i}, 57 \%$, dr $2: 1^{\mathrm{b}}$<smiles>CC(C)NC(=O)C1N(C(=O)c2ccco2)c2ccccc2C1(C)C</smiles>

$3 \mathbf{j}, 35 \%$

${ }^{a}$ Conditions: amine $(0.50 \mathrm{mmol})$, carboxylic acid (1.5 eq.), isocyanide (1.5 eq.), IBX (1 eq.), DCM $(0.5 \mathrm{M}), 60^{\circ} \mathrm{C}, 48 \mathrm{~h}$, closed vessel. Isolated yield. Only one diastereoisomer observed, unless stated otherwise. ${ }^{b}$ Ratio determined by NMR analysis of the crude mixture. ${ }^{22}$
For the majority of examples, a single diastereoisomer of the dipeptide was isolated $(\mathbf{3 a}-\mathbf{h})$. However, exo-configured dipeptide $3 \mathbf{i}$ was obtained as a mixture of diastereoisomers (Fig. 2). ${ }^{22}$ The increased diastereoselectivity with endo-configured

a)
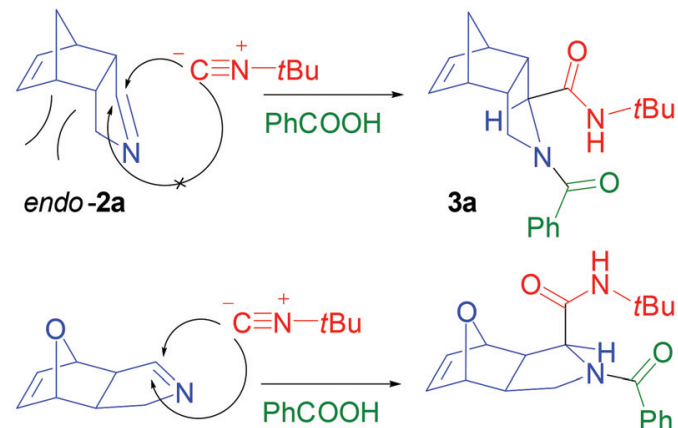

exo-2c
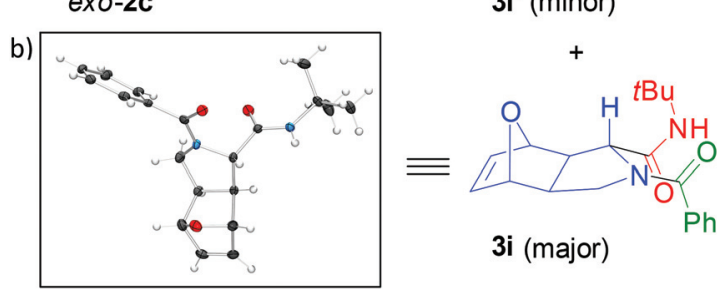

Fig. 2 (a) Steric rationalization for superior diastereoselectivity for the Ugi-type reaction of endo-configured 2a compared to exo-2c. (b) Plot of the molecular structure of major diastereoisomer $3 \mathbf{i}$ with displacement ellipsoids drawn at $50 \%$ probability. $\dagger$

Table 5 Oxidative aza-Friedel-Crafts reaction ${ }^{a}$

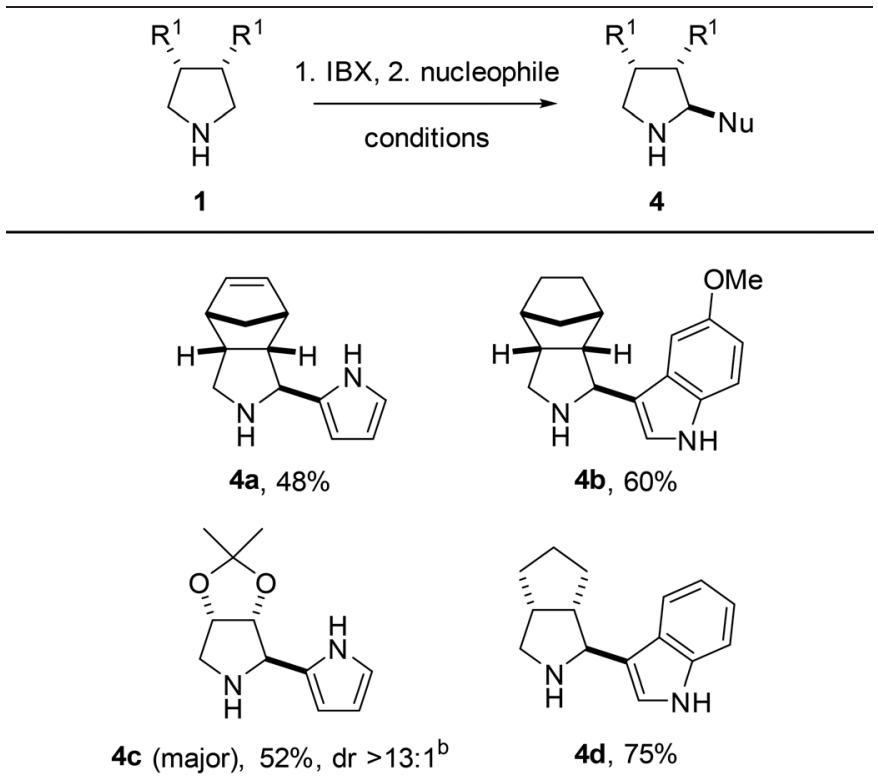

${ }^{a}$ Conditions: 1 . amine $(0.25 \mathrm{mmol})$, IBX (1 eq.), DCM $(0.2 \mathrm{M}), 60{ }^{\circ} \mathrm{C}$, $1 \mathrm{~h}$; 2 . nucleophile (2.0 eq.), TFA ( 2.0 eq.), $60^{\circ} \mathrm{C}, 1 \mathrm{~h}$, closed vessel. Isolated yield. Only a single diastereoisomer was observed, unless stated otherwise. ${ }^{b}$ Diastereoisomers were inseparable; ratio determined by NMR analysis of the crude mixture. 
imines ${ }^{11 a}$ such as $2 \mathbf{a}$ is explained by steric congestion on the concave face of the molecule, facilitating selective attack of the nucleophile from the other side (Fig. 2a). For exo-configured 2c, the steric effect is expected to be less pronounced.

In order to expand the molecular diversity available by reactions of in situ-generated bicyclic pyrrolines, we decided to explore the aza-Friedel-Crafts reaction. ${ }^{23}$ Our one-pot methodology showed to be effective in an oxidative aza-Friedel-Crafts reaction with pyrrole and indoles as a two-step procedure, giving 2-substituted pyrrolidines in modest to good yield (Table 5, 48-75\%) and high to excellent diastereoselectivity. The yield of pyrrolidine $\mathbf{4 c}$ even exceeded the yield of its hemiaminal intermediate $2 \mathbf{f}$, supporting our hypothesized instability of the latter species. Unfortunately, 6-nitro- and 3-methylsubstituted indoles were not suitable reaction partners, presumably as a result of their reduced nucleophilicity.

In conclusion, we have developed the first IBX-mediated oxidation of unactivated aliphatic amines to give 1-pyrrolines. Moreover, an efficient one-pot protocol for diastereoselective $\alpha$-functionalization of meso-pyrrolidines in oxidative Ugi-type and aza-Friedel-Crafts reactions is presented.

\section{Acknowledgements}

The Netherlands Organization of Scientific Research (NWO) is gratefully acknowledged for financial support by means of a TOP grant. The authors thank Christophe Vande Velde (University of Antwerp, Belgium) for the X-ray structure determination, and Matthias Zeller (Youngstown State University, USA) for the collection of the X-ray diffraction data. The diffractometer was funded by NSF Grant 1337296. We thank Elwin Janssen for technical assistance, Sanne Bouwman for HRMS measurements, and Dr Andreas W. Ehlers for NMR maintenance.

\section{Notes and references}

1 For selected reviews, see: (a) T. Wirth, Angew. Chem., Int. Ed., 2005, 44, 3656; (b) V. V. Zhdankin and P. J. Stang, Chem. Rev., 2008, 108, 5299; (c) T. Dohi and Y. Kita, Chem. Commun., 2009, 2073; (d) V. V. Zhdankin, J. Org. Chem., 2011, 76, 1185; (e) L. F. Silva and B. Olofsson, Nat. Prod. Rep., 2011, 28, 1722; (f) A. Parra and S. Reboredo, Chem. Eur. J., 2013, 19, 17244.

2 For selected reviews, see: (a) U. Ladziata and V. V. Zhdankin, ARKIVOC, 2006, 26; (b) V. Satam, A. Harad, R. Rajule and H. Pati, Tetrahedron, 2010, 66, 7659; (c) A. Duschek and S. F. Kirsch, Angew. Chem., Int. Ed., 2011, 50, 1524.

3 M. Frigerio, M. Santagostino and S. Sputore, J. Org. Chem., 1999, 64, 4537.

4 (a) K. C. Nicolaou, C. J. N. Mathison and T. Montagnon, Angew. Chem., Int. Ed., 2003, 42, 4077; (b) K. C. Nicolaou, C. J. N. Mathison and T. Montagnon, J. Am. Chem. Soc.,
2004, 126, 5192; (c) S. N. Murthy and Y. V. D. Nageswar, Tetrahedron Lett., 2011, 52, 4481.

5 A single case of oxidation at a nonactivated position was unexpectedly observed for $\mathrm{N}$-methylbenzylamine; see ref. $4 a$.

6 For selected examples of the oxidation of unactivated secondary amines to the corresponding imine with other oxidants including hypervalent iodine reagents, see: (a) S. Furukawa, A. Suga and T. Komatsu, Chem. Commun., 2014, 50, 3277; (b) T. Sonobe, K. Oisaki and M. Kanai, Chem. Sci., 2012, 3, 3249; (c) V. Köhler, K. R. Bailey, A. Znabet, J. Raftery, M. Helliwell and N. J. Turner, Angew. Chem., Int. Ed., 2010, 49, 2182; (d) B. Zhu and R. J. Angelici, Chem. Commun., 2007, 2157; (e) X.-Q. Gu, W. Chen, D. Morales-Morales and C. M. Jensen, J. Mol. Catal. A: Chem., 2002, 189, 119; (f) S. Minakata, Y. Ohshima, A. Takemiya, I. Ryu, M. Komatsu and Y. Ohshiro, Chem. Lett., 1997, 311; $(g)$ M. Ochiai, D. Kajishima and T. Sueda, Heterocycles, 1997, 46, 71; (h) J. Larsen and K. A. Jørgensen, J. Chem. Soc., Perkin Trans. 2, 1992, 1213; (i) M. Ochiai, M. Inenaga, Y. Nagao, R. M. Moriarty, R. K. Vaid and M. P. Duncan, Tetrahedron Lett., 1988, 29, 6917; (j) H. B. Henbest and P. Slade, J. Chem. Soc., 1960, 1558.

7 For selected reviews, see: (a) C. J. Li, Acc. Chem. Res., 2009, 42, 335; (b) E. A. Mitchell, A. Peschiulli, N. Lefevre, L. Meerpoel and B. U. W. Maes, Chem. - Eur. J., 2012, 18, 10092.

8 For a selected review, see: S. A. Girard, T. Knauber and C. J. Li, Angew. Chem., Int. Ed., 2014, 53, 74.

9 P. Fontaine, A. Chiaroni, G. Masson and J. Zhu, Org. Lett., 2008, 10, 1509.

10 (a) T. Ngouansavanh and J. P. Zhu, Angew. Chem., Int. Ed., 2006, 45, 3495; (b) F. De Moliner, S. Crosignani, A. Galatini, R. Riva and A. Basso, ACS Comb. Sci., 2011, 13, 453.

11 For an IBX-mediated oxidative Ugi four-component reaction, see: (a) F. Drouet, G. Masson and J. P. Zhu, Org. Lett., 2013, 15, 2854; For IBX-mediated oxidative Ugi-type threecomponent reactions, see: (b) T. Ngouansavanh and J. Zhu, Angew. Chem., Int. Ed., 2007, 46, 5775; (c) D. Zhu, L. Xia, L. Pan, S. Li, R. Chen, Y. Mou and X. Chen, J. Org. Chem., 2012, 77, 1386.

12 (a) A. Znabet, E. Ruijter, F. J. J. de Kanter, V. Kohler, M. Helliwell, N. J. Turner and R. V. A. Orru, Angew. Chem., Int. Ed., 2010, 49, 5289; (b) A. Znabet, M. M. Polak, E. Janssen, F. J. J. de Kanter, N. J. Turner, R. V. A. Orru and E. Ruijter, Chem. Commun., 2010, 46, 7918; (c) A. Znabet, J. Zonneveld, E. Janssen, F. J. J. De Kanter, M. Helliwell, N. J. Turner, E. Ruijter and R. V. A. Orru, Chem. Commun., 2010, 46, 7706; (d) A. Znabet, S. Blanken, E. Janssen, F. J. J. de Kanter, M. Helliwell, N. J. Turner, E. Ruijter and R. V. A. Orru, Org. Biomol. Chem., 2012, 10, 941.

13 Standard procedures for the oxidation of aliphatic amines to imines are performed using a two-step protocol of $N$-halogenation followed by base-mediated elimination. Unfortunately, we experienced these procedures to be 
highly dependent on concentration and batch of halogenation reagent and therefore difficult to reproduce.

14 The oxidation was amenable to scale up $(4 \mathrm{mmol})$ with minor changes to the general procedure $(0.4 \mathrm{M}, 2 \mathrm{~h})$, affording imine $2 \mathrm{a}$ in $76 \%$ yield.

15 T. M. Chapman, S. Courtney, P. Hay and B. G. Davis, Chem. - Eur. J., 2003, 9, 3397.

16 Piperidine derivatives and acyclic secondary amines were not converted under these conditions.

17 For selected reviews, see: (a) C. de Graaff, E. Ruijter and R. V. A. Orru, Chem. Soc. Rev., 2012, 41, 3969; (b) A. Dömling, W. Wang and K. Wang, Chem. Rev., 2012, 112, 3083; (c) G. van der Heijden, E. Ruijter and R. V. A. Orru, Synlett, 2013, 666; (d) R. C. Cioc, E. Ruijter and R. V. A. Orru, Green Chem., 2014, 16, 2958.

18 For selected reviews, see: (a) A. Dömling and I. Ugi, Angew. Chem., Int. Ed., 2000, 39, 3169; (b) A. Dömling, Chem. Rev., 2006, 106, 17.

19 For the corresponding diastereoselective Ugi-type reaction from 1-pyrrolines, see ref. 11a. For examples of photoredox, aerobic and transition metal-catalyzed oxidative Ugi-type three component reactions with either carboxylic acid or water, see (a) Y. Chen and G. Feng, Org. Biomol. Chem., 2015, 13, 4260; (b) S. U. Dighe, S. Kolle and S. Batra, Eur. J. Org. Chem., 2015, 4238; (c) C. Xie and L. Han, Tetra- hedron Lett., 2014, 55, 240; (d) C. Vila and M. Rueping, Green Chem., 2013, 15, 2056; (e) X. Ye, C. Xie, R. Huang and J. Liu, Synlett, 2012, 409; $(f)$ G. Jiang, J. Chen, J.-S. Huang and C.-M. Che, Org. Lett., 2009, 11, 4568.

20 K. C. Nicolaou, T. Montagnon, P. S. Baran and Y. L. Zhong, J. Am. Chem. Soc., 2002, 124, 2245.

21 The oxidation of dihydroindoline and tetrahydroquinoline was only selective when using an excess of IBX (2 eq.) and a stoichiometric amount of radical inhibitor TEMPO (1-1.5 eq.), providing the aromatized products. This observation supports previous hypotheses by Nicolaou et al. that the oxidation of amines with IBX proceeds via an ionic mechanism, while benzylic oxidation towards acetophenone derivatives follows a single electron transfer (SET) mechanism. TEMPO itself does not facilitate the oxidation.

22 The relative configurations of the major diastereoisomer $\mathbf{3 i}$, determined by X-ray crystallography, and minor diastereoisomer $3 \mathbf{i}^{\prime}$ are depicted in Figure 2.

23 For examples of transition metal-catalyzed oxidative azaFriedel-Crafts reactions with a stoichiometric oxidant, see ref. $6 b$ and references therein, as well as: $(a)$ A. J. Catino, J. M. Nichols, B. J. Nettles and M. P. Doyle, J. Am. Chem. Soc., 2006, 128, 5648; (b) Z. Li, D. S. Bohle and C.-J. Li, Proc. Natl. Acad. Sci. U. S. A., 2006, 103, 8928; (c) Z. Li and C.-J. Li, J. Am. Chem. Soc., 2005, 127, 6968. 